\title{
TRATAMIENTO DE LA ENFERMEDAD HEPÁTICA GRASA NO ALCOHÓLICA
}

\author{
TREATMENT OF NON-ALCOHOLIC FATTY LIVER DISEASE
}

\author{
Susana Gutt ${ }^{1}$, María Álvarez², María Yuma ${ }^{3}$, Marianela Ackermann ${ }^{4}$, María Coronel $^{5}$, Carla Gauna ${ }^{6}$, \\ Sandra González ${ }^{7}$, Paula Lifszyc ${ }^{8}$, Estrella Menéndez ${ }^{9}$, Juliana Mociulsky ${ }^{10}$, Carla Musso ${ }^{11}$, Paola Polo ${ }^{12}$, \\ Victoria Salinas ${ }^{13}$, Guadalupe Vanoli ${ }^{14}$, Susana Fuentes ${ }^{15}$
}

\section{RESUMEN}

La enfermedad del hígado graso no alcohólico (EHGNA) es una enfermedad del hígado que no se asocia con el alcohol. Su prevalencia aumenta junto con la epidemia de obesidad y diabetes tipo 2 (DM2), y el riesgo de sufrir una enfermedad hepática más agresiva es mayor con el consiguiente aumento de la cirrosis, el carcinoma hepatocelular y la enfermedad cardiovascular (ECV). La acumulación ectópica de grasa se asocia a dietas hipergrasas y de alta densidad energética, a la hiperglucemia y la insulinorresistencia que condicen a la supresión de la producción hepática de glucosa y la disminución de la captación de glucosa por el músculo esquelético lo cual promueve la lipogénesis de novo y genera un círculo vicioso que favorece aún más la esteatosis. La implementación de estrategias de prevención y tratamiento constituye el desafío actual. El tratamiento de EHGNA incluye el descenso del peso corporal en lo posible a rangos saludables, un buen control de la diabetes y de otros factores de riesgo cardiovascular. Varios fármacos fueron probados, pero aún ninguno aprobado específicamente para esteatohepatitis no alcohólica (EHNA). La cirugía bariátrica se considera una posibilidad terapéutica en pacientes obesos que tienen enfermedad hepática como comorbilidad, y sugieren que la presencia de EHGNA o EHNA no contraindica la cirugía incluso favorece la disminución de la probabilidad de desarrollar cirrosis en pacientes con alto riesgo.

Palabras clave: estatosis, diabetes e hígado graso, hígado graso y farmacoterapia, cirugía bariátrica e hígado graso.

Revista de la Sociedad Argentina de Diabetes 2018; Vol. 52 (10-24)

\section{ABSTRACT}

Nonalcoholic fatty liver disease (NAFLD) is a fatty disease of the liver that is not associated with alcohol. Its prevalence increases along with the epidemic of obesity and type 2 diabetes, and the risk of suffering a more aggressive liver disease is greater with the consequent increase in cirrhosis, hepatocellular carcinoma and cardiovascular disease (CVD). The ectopic accumulation of fat is associated with high-fat diets and high energy density, hyperglycemia and insulin resistance that lead to the suppression of hepatic glucose production and decreased glucose uptake by the skeletal muscle which promotes the lipogenesis of novo and generates a vicious circle that favors even more steatosis.

The implementation of prevention and treatment strategies constitutes the current challenge. Treatment of NAFLD includes decreasing body weight as much as possible to healthy ranges, good control of diabetes and other cardiovascular risk factors. Several drugs were tested, but none were approved specifically for non alcoholic steatohepatitis (NASH).

Bariatric surgery is considered a therapeutic possibility in obese patients who have liver disease as comorbidity, and suggest that the presence of NAFLD or NASH does not contraindicate surgery even favors the decrease in the probability of developing cirrhosis in patients with high risk.

Key words: non alcoholic fatty liver disease, steatosis, diabetes and fatty liver, fatty liver pharmacotherapy, nariatric surgery and fatty liver.

Revista de la Sociedad Argentina de Diabetes 2018; Vol. 52 (10-24)
1 Médica especialista en Nutrición, Jefa de la Sección Nutrición, Servicio de Clínica Médica, Hospital Italiano de Buenos Aires, Ciudad Autónoma de Buenos Aires, Argentina

2 Médica especialista en Nutrición, Coordinadora del Centro de Estudio y Tratamiento de la Obesidad Severa-CETOS, Ciudad Autónoma de Buenos Aires, Argentina

3 Médica especialista en Nutrición, Magister en Diabetes; Médica de Planta Sección Nutrición, Servicio de Clínica Médica Hospital Italiano de Buenos Aires, Ciudad Autónoma de Buenos Aires, Argentina

4 Médica especialista en Medicina Interna y Nutrición, Magister en Diabetes; Directora del área Diabetes y Nutrición Centro Integral de Endocrinología y Nutrición, Corrientes, Argentina

5 Médica especialista en Medicina Interna, Médica del Centro Médico Maffei, Ciudad Autónoma de Buenos Aires, Argentina

6 Médica especialista en Medicina Interna, Médica de Planta del Servicio de Diabetología del Hospital de Clínicas "José de San Martín", Ciudad Autónoma de Buenos Aires, Argentina
7 Médica del Consultorio de Diabetes, Clínica IMA, Provincia de Buenos Aires, Adrogué, Argentina

8 Médica especialista en Nutrición, Médica de Planta del Servicio de Nutrición Hospital General de Agudos "Dr. Carlos Durand", Ciudad Autónoma de Buenos Aires, Argentina

9 Médica especialista en Nutrición, Médica de Planta del Servicio de Nutrición y Diabetes Centro de Educación Médica e Investigaciones Clínicas "Dr. Norberto Quirno" (CEMIC), Ciudad Autónoma de Buenos Aires, Argentina

10 Médica Endocrinóloga, Jefa de la Unidad Nutrición, Instituto Cardiovascular Buenos Aires, ICBA, Ciudad Autónoma de Buenos Aires, Argentina

11 Médica Endocrinóloga, Coordinadora de Diabetes, Fundación Favaloro; Médica de Planta de la Unidad Asistencial "César Milstein"- Instituto Nacional de Servicios Sociales para Jubilados y Pensionados, Ciudad Autónoma de Buenos Aires, Argentina

12 Médica Endocrinóloga Sanatorio Las Lomas; Médica de Planta del Servicio de Endocrinología Hospital San José de la Ciudad de Campana, Provincia de Buenos Aires, Argentina 
13 Médica especialista en Clínica y Nutrición, integrante del Departamento de Clínica Médica y Nutrición de CEMIC, Ciudad Autónoma de Buenos Aires, Argentina

14 Médica especialista en Nutrición, Médica de Planta de la Unidad de Nutrición y Diabetes del Hospital General de Agudos "José María Ramos Mejía", Ciudad Autónoma de Buenos Aires, Argentina

15 Médica especialista en Medicina Interna, Médica del Hospital "El Cruce", Servicio de Atención Médica Integral para la Comunidad, Provincia de Buenos Aires, Argentina

${ }^{1-15}$ Comité Obesidad y Cirugía Bariátrica de la Sociedad Argentina de Diabetes, Ciudad Autónoma de Buenos Aires, Argentina
Contacto de la autora: Susana Gutt

E-mail: susana.gutt@gmail.com

Correspondencia: Pte. J.E. Uriburu 1076 PB "4", (1114AAF),

Ciudad Autónoma de Buenos Aires, Argentina

Tel.: ( 011) 4822-5181. Celular: (011) 1544267394

Fecha de trabajo recibido: 27/03/2018

Fecha de trabajo aceptado: 06/04/2018

Conflictos de interés: las autoras declaran que no existe conflicto de interés

\section{INTRODUCCIÓN}

La enfermedad hepática grasa no alcohólica (EHGNA) es una enfermedad por depósito de grasa en el hígado que no se asocia al alcohol; incluye distintos estadios: desde una simple esteatosis, la esteatohepatitis no alcohólica (EHNA), la fibrosis hepática y puede llegar a la cirrosis ${ }^{1,2}$.

Su prevalencia aumenta en paralelo al avance de la obesidad y la diabetes tipo 2 (DM2), y actualmente constituye la enfermedad hepática crónica más frecuente. Es considerada la manifestación hepática del síndrome metabólico (SM) y de la insulinorresistencia $(\mathrm{RI})^{3,4}$.

La prevalencia de EHGNA en la población general se estima entre un 10 y $30 \%$; en pacientes con DM2 aumenta a más del $76 \%$ y en obesos severos a más del $90 \%{ }^{5}$. En Latinoamérica su prevalencia e incidencia se desconoce?

La EHGNA se define por la presencia de grasa en $\geq 5 \%$ de los hepatocitos en estudios histológicos y más de 5,6\% por técnicas de resonancia magnética (RNM) en ausencia de otras enfermedades hepáticas con etiología conocida como las enfermedades virales o autoinmunes, toxicidad hepática por fármacos como la amiodarona, ácido valproico o el tamoxifeno, hemocromatosis, enfermedad de Wilson o consumo de riesgo de alcohol5,6.

La etiología de la EHNA es multifactorial; involucra al tejido adiposo (TA) disfuncional e insulinorresistente que favorece el depósito ectópico de los triglicéridos en diferentes tejidos, lo cual es más evidente en pacientes con $\mathrm{DM}^{7}$.

Clínicamente los pacientes que presentan EHGNA tienden a ser obesos con resistencia a la insulina (RI) y/o DM2, dislipidemia (DLP) e hipertensión arterial (HTA), todos factores de riesgo para enfermedad cardiovascular ateroesclerótica (ECVA).

Para el año 2040 la prevalencia de DM2 será de 642 millones de adultos en el mundo; la EHGNA acompañará dicho aumento y el riesgo de padecer una enfermedad hepática más agresiva es mayor, con el consiguiente aumento de cirrosis, carcinoma hepatocelular y ECVA? . Por ello la implementación de estrategias de prevención y tratamiento constituye el actual desafío.

\section{Patogénesis}

La EHGNA es el resultado de la interacción de diversos factores que contribuyen a su aparición. Las dietas de alto valor calórico, con exceso de grasas saturadas e hidratos de carbono refinados, bebidas azucaradas y la alta ingesta de jarabe de maíz alta fructosa en la dieta occidental se asocia a obesidad, DM2 y EHGNA ${ }^{8}$.

En este contexto la Rl y la acumulación de ácidos grasos libres (AGL) provenientes de la lipólisis delTA, al cual corresponde el $60-70 \%$ de los lípidos que llega al hígado cuyo efecto lipotóxico produce una acumulación de grasa en tejidos que bajo condiciones normales no se comportan como depósitos grasos (por ejemplo, hígado, músculo esquelético, páncreas) promueven la disfunción o muerte celular. Los AGL también provienen de la síntesis hepática por exceso de hidratos de carbono (lipogénesis de novo) o de los triglicéridos (TG) dietarios ${ }^{2-8}$.

La acumulación ectópica de grasa se asocia a hiperglucemia, insulinorresistencia, supresión de la producción hepática de glucosa y disminución de la captación de glucosa por el músculo esquelético lo cual promueve la lipogénesis de novo y genera un círculo vicioso que favorece aún más la esteatosis.

Se han identificado muchas variantes genéticas, si bien la mejor asociación genética caracterizada es la PNPLA3 y recientemente el genTM6SF2 se relacionó con la EHGNA. La genotipificación no está considerada de rutina en la EHGNA. 


\section{DIAGNÓSTICO}

Las dificultades para su diagnóstico se deben a que el estudio histológico (la biopsia hepática) es el único método para determinar con certeza la presencia de esteatosis, determinar la actividad inflamatoria, estadificar la fibrosis y efectuar el diagnóstico diferencial con otras entidades, pero su realización diagnóstica en todos los pacientes no está indicada para el tamizaje ni el seguimiento por ser un procedimiento invasivo de riesgo moderado. Se considera el método diagnóstico "gold standard" o "patrón oro".

Dado que la enfermedad grasa del hígado abarca desde la esteatosis leve, la esteatosis con inflamación lobular o portal sin balonamiento y la esteatosis con balonamiento pero sin inflamación, el diagnóstico de la esteatohepatitis no alcohólica (EHNA) requiere demostrar la inflamación, el balonamiento y la esteatosis hepática.

\section{Diagnóstico clínico o no invasivo}

En la atención primaria la presencia de factores de riesgo como obesidad, DM2, DLP, RI e HTA debe sugerir la presencia de EHGNA aún sin aumento de las enzimas hepáticas lo cual obliga a descartar otras causas de esteatosis hepática con pruebas de función hepática que excluyan otros desencadenantes de hepatitis.

A nivel de la atención especializada deben identificarse aquellos con peor pronóstico, por ejemplo EHNA severa, evaluar la progresión de la enfermedad y monitorear la evolución en función de la respuesta a las intervenciones terapéuticas. El logro de estos objetivos reduciría la necesidad de biopsia hepática ${ }^{1-8}$.

Los estudios de laboratorio permitirán aproximar el diagnóstico de SM, con alteraciones en la glucemia, dislipidemia con fenotipo aterogénico (TG elevados y HDL baja) ya que debe tenerse en cuenta que el $80 \%$ de los pacientes con EHGNA tiene las concentraciones séricas de las enzimas hepáticas dentro de los límites normales, independientemente de si se trata de una esteatosis simple o una esteatohepatitis, excepto en aquellos con una cirrosis establecida los cuales pueden presentar elevación de las transaminasas y de la bilirrubina, así como un descenso de la albúmina, del tiempo de protrombina y de las plaquetas.

\section{Diagnóstico por imágenes}

El aumento de depósito graso a nivel hepático puede observarse con ecografía, tomografía computarizada o resonancia magnética (RNM), sin embargo estas imágenes no permiten diferenciar la esteatosis simple de la esteatohepatitis con o sin fibrosis.

La espectroscopia protónica por resonancia magnética y la elastografía transitoria parecen ser más útiles para valorar la esteatosis y la fibrosis respectivamente, pero aún deben ser validadas mediante estudios clínicos en grandes poblaciones de pacientes antes de su implementación en la práctica clínica.

\section{Biopsia hepática}

El estudio histológico es considerado el "patrón de oro" para constatar los cambios de la célula hepática, la presencia de inflamación (esteatohepatitis) o de fibrosis y determinar la evolución hacia la cirrosis.

La biopsia hepática puede evidenciar la presencia de macro y microvacuolas, con desplazamiento del núcleo en $\geq$ del $5 \%$ de los hepatocitos, balonización, inflamación portal y perisinusoidal, cuerpos acidófilos, megamitocondrias, acumulación de glucógeno en los núcleos con fibrosis porto-portal, porto centrolobulillar o perisinusoidal'.

El Instituto Nacional de Diabetes, Enfermedades Digestivas y Renales desarrolló un puntaje de actividad de EHGNA denominado NAS utilizado para la clasificación histopatológica ${ }^{9}$ (Tabla 1).

\begin{tabular}{|c|c|c|c|}
\hline Puntaje & Esteatosis & $\begin{array}{c}\text { Inflamación } \\
\text { lobulillar }\end{array}$ & Balonamiento \\
\hline 0 & $<5 \%$ & No & No \\
\hline 1 & $5-33 \%$ & $<2$ & Pocas células \\
\hline 2 & $34-65 \%$ & $2-4$ & Prominente \\
\hline 3 & $\geq 66 \%$ & $>4$ & Prominente \\
\hline
\end{tabular}

Tabla 1: Cambios histopatológicos en la clasificacion de NAS.

Las imágenes histológicas refieren a la biopsia hepática para diagnóstico de EGHNA y EHNA. Se observa la infiltración grasa, el balonamiento de los hepatocitos, la esteatosis mixta con fibrosis perisinusoidal y finalmente cuando la esteatohepatitis ha dañado el hígado y sobreviene la etapa final con fibrosis (Figuras 1, 2, 3, 4 y 5. Imágenes histológicas de biopsia hepática publicadas con la autorización del Dr. Sebastián Marciano). 


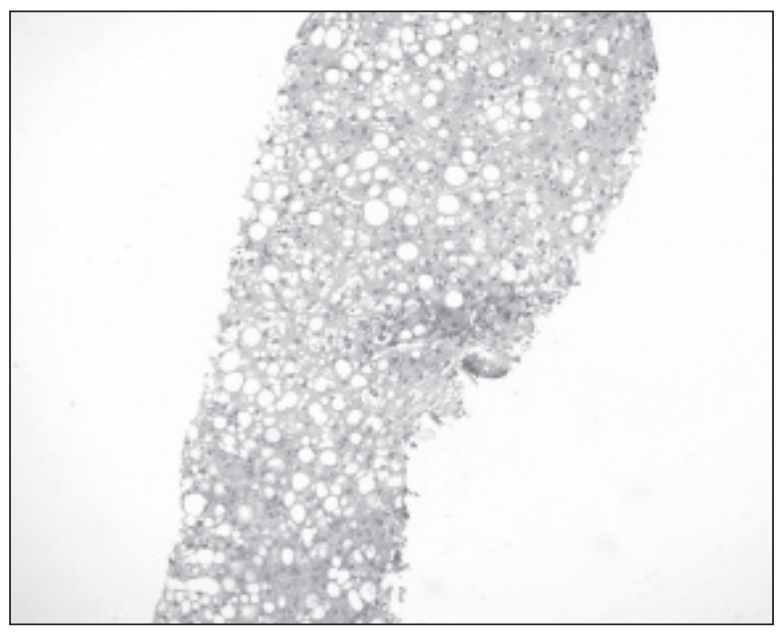

Figura 1: Biopsia hepática: esteatosis mixta de gotas grandes y pequeñas sin lesión hepática significativa (tinción con hematoxilina y eosina, 100X).

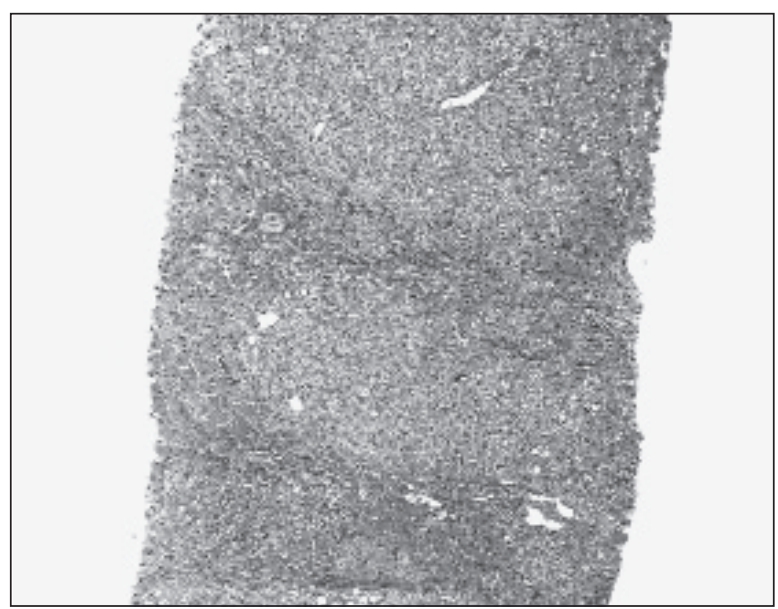

Figura 2: Biopsia hepática: esteatosis mixta con fibrosis perisinusoidal delicada (tinción deTrichrome, 100X).

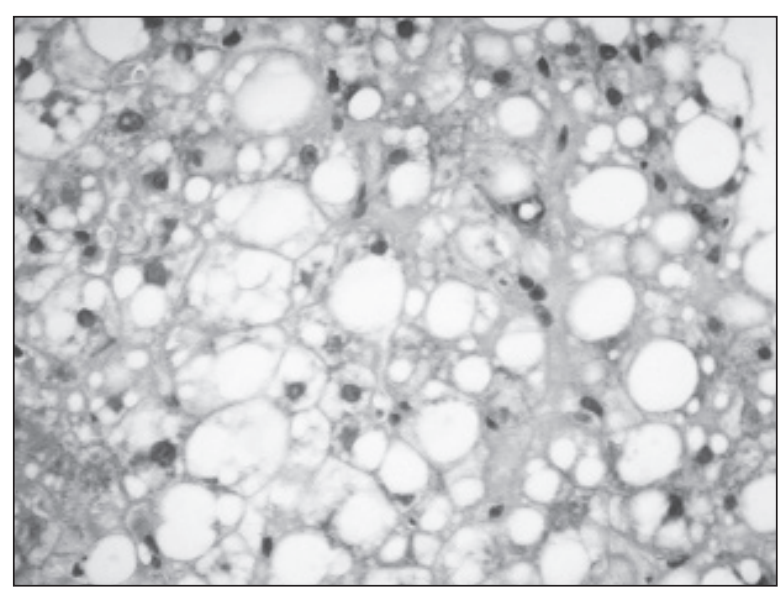

Figura 3: Biopsia hepática: esteatosis mixta con lesión hepática. Hepatocitos con globo con cuerpos de Mallory-Denk y actividad inflamatoria parenquimatosa leve (tinción con hematoxilina y eosina, 400X).

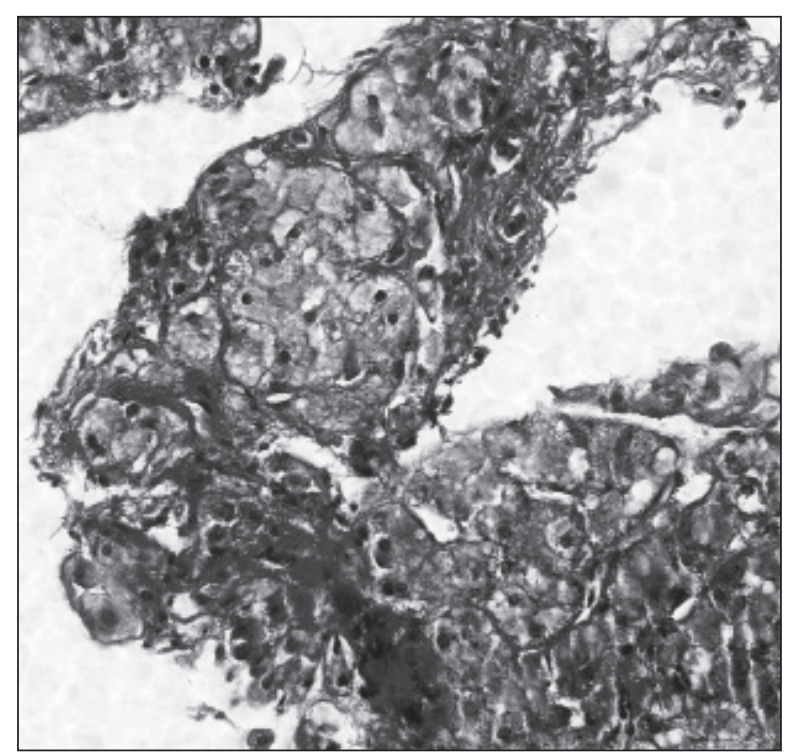

Figura 4: Biopsia hepática: EHNA calcinada en la etapa final. Fibrosis parenquimatosa sin esteatosis hepática (tinción con tricrómico, 400X).

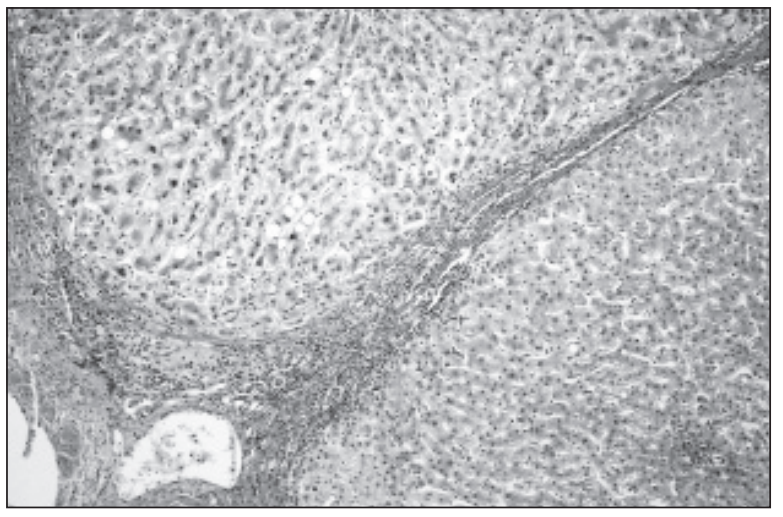

Figura 5: Cirrosis macronodular debido a EHNA quemada. Pocos hepatocitos con esteatosis macrovesicular cerca de grandes septos fibrosos con actividad inflamatoria mixta (tinción con tricrómico, 100X).

\section{TRATAMIENTO}

La EHGNA y EHNA son enfermedades que se presentan con frecuencia en poblaciones con obesidad y DM2, y según Cusi Kenneth ${ }^{5}$, la magnitud de la epidemia hace imperativa la evaluación especialmente en esta población de riesgo. Los pacientes con DM2 que también tienen EHNA presentan un mayor riesgo de muerte por cirrosis, hepatocarcinoma o enfermedad cardiovascular. Actualmente debe intervenirse en la EHGNA igual que se hace con las complicaciones microvasculares de la diabetes ${ }^{2-5}$. El mejor tratamiento disponible en la actualidad para la mejoría de la 
EHGNA es el descenso de peso, ya sea por cambios en el estilo de vida, fármacos o a través de la cirugía bariátrica $(\mathrm{CB})^{10}$. La CB ha demostrado ser el tratamiento más efectivo para lograr un descenso de peso significativo y sostenido en el largo plazo. Junto con este beneficio se observa la mejoría de otros componentes del síndrome metabólico, de la hipertensión arterial, de los trastornos del metabolismo glucémico y lipídico, incluso de la EHGNA ${ }^{11}$.

\section{Dieta y cambio de estilo de vida}

Las modificaciones en el estilo de vida del paciente, principalmente dieta y actividad física, son muy importantes en relación a la EHGNA. Una reducción de peso del 5 al 10\% sumado a la actividad física puede disminuir el $40 \%$ de los niveles de TG intrahepáticos.

Un descenso del $10 \%$ del peso corporal con modificación del estilo de vida logra mejoría de la esteatosis, la necrosis y la inflamación en pacientes con EHNA diagnosticada por biopsia hepática. Algunos autores refieren que la fibrosis no mejora con esta intervención ni aún con la cirugía bariátrica ${ }^{2,6,8}$, mientras otros sugieren que con un descenso $\geq$ al $10 \%$ del peso corporal la fibrosis puede revertirse hasta en un $45 \%$.

La evidencia demuestra que con un descenso $\geq$ al $7 \%$ del peso corporal hay mejoría de score de actividad de la EHNA en un $64-90 \%$ de los casos, con un descenso $\geq$ al $5 \%$ del peso corporal revierte el balonamiento y la inflamación en un $41-100 \%$, y con un descenso $\geq$ al $3 \%$ del peso corporal la esteatosis hepática se resuelve en un $35-100 \%$ de los casos ${ }^{12}$.

En pacientes con obesidad la dieta debe contemplar una restricción calórica de aproximadamente el $30 \%$ de los requerimientos, con supresión de alimentos y bebidas con alto contenido de fructosa. No hay suficiente evidencia que indique suplementar la dieta con ácidos grasos poliinsaturados.

\section{EFECTOS SOBRE LA EHGNA DE LOS AGENTES FAR- MACOLÓGICOS PARA ELTRATAMIENTO DE LA DM2}

\section{Agentes sensibilizadores de la insulina}

La insulinorresistencia es un mecanismo importante en el desarrollo y progresión de la EHGNA, de este modo la investigación se ha centrado en el potencial efecto terapéutico de los sensibilizadores de insulina en esta enfermedad ${ }^{13 .}$

\section{Metformina}

La metformina (MTF), compuesto de la clase farmacológica de las biguanidas con conocidos efectos beneficiosos sobre la resistencia a la insulina, contribuye a la disminución de la glucosa en la sangre al reducir la gluconeogénesis hepática, inducir la captación de glucosa por los músculos y aumentar la oxidación de los ácidos grasos en el tejido adiposo. El principal mediador molecular mediante el cual la MTF actúa para mejorar la EHNA es por activación de la vía de la proteína quinasa dependiente del monofosfato de adenosina $(\mathrm{AMP}-\mathrm{K})^{14}$.

Un metaanálisis que incluyó datos publicados de estudios clínicos controlados concluyó que luego de 6-12 meses de tratamiento con MTF asociada a cambios en el estilo de vida no mejoró la histología hepática ni los niveles de aminotransferasas en comparación con los cambios en el estilo de vida solamente, independientemente de la dosis, duración del tratamiento o el estado diabético $^{15}$. La MTF mejora la sensibilidad a la insulina y los niveles en suero de la TGP y de la aspartato aminotransferasa en la mayoría de los sujetos; sin embargo no tiene efecto significativo sobre la histología del hígado. La dosis exacta y la duración del tratamiento son desconocidos y los efectos beneficiosos en el suero sobre la TGP sólo continúan durante el tratamiento ${ }^{16}$.

La Asociación Americana para el Estudio de las Enfermedades del Hígado (AASLD, Association for the Study of Liver Diseases) no recomienda la MTF como un tratamiento específico para la EHNA. Una declaración de posición sobre EHNA/ NASH basada en una conferencia especial de la European Association for the Study of the Liver (EASL) en el año 2010 tampoco recomienda la MTF para la terapia específica dirigida al hígado de $\mathrm{NASH}^{17,18}$. La guía de la AASLD en el año 2012 para el diagnóstico y el tratamiento del EHNA considera que la MTF que no tiene efecto significativo en la histología hepática y no se aconseja como un tratamiento específico de EHNA ${ }^{17}$. Sin embargo, teniendo en cuenta que los efectos beneficiosos de la MTF sobre la resistencia a la insulina están bien establecidos y que la EHNA se relaciona estrechamente con la insulinorresistencia, la MTF puede utilizarse para el manejo de la EHGNA/ EHNA en pacientes con diabetes o resistencia a la insulina ${ }^{7,19}$. 


\section{Tiazolidinedionas}

Las tiazolidinedionas (TZD) o "glitazonas" son agentes orales insulinosensibilizadores para el tratamiento de la DM2 que actúan sobre la insulinorresistencia y la inflamación favorecida por la lipotoxicidad hepática en la EHNA. Existe consenso en que su mecanismo de acción principal es la activación del receptor peroxisomal gamma (PPAR $\gamma$ ) cuyos receptores nucleares se encuentran principalmente en TA, hígado, macrófagos y otras células inmunes, célula beta pancreática, hueso y otros tejidos.

Las TZD son ligandos del factor de transcripción de los receptores PPAR $\gamma$ y es en el tejido hepático donde existe la mayor cantidad de estos receptores. La pioglitazona aumenta la expresión de genes implicados en la adipogénesis y en la oxidación de ácidos grasos y estimula la secreción de adiponectina en las células adiposas. Esta última, a su vez, activa la AMPK hepática y muscular que estimula la oxidación de ácidos grasos e inhibe la excesiva síntesis de ácidos grasos hepáticos. Las TZD tienen amplios efectos en el metabolismo de la glucosa por modulación del sustrato, señalización de la insulina y oxidación del ácido graso mitocondrial? ${ }^{7}$ Se ha evaluado el efecto de este grupo de drogas con resultados beneficiosos sobre la esteatosis, la balonización hepática y la inflamación pero existen pocos estudios que demuestren mejoría de la fibrosis. Un metaanálisis de cuatro ensayos aleatorizados que compararon tiazolidinedionas vs placebo en 334 pacientes con EHNA demostró que las TZD mejoran los parámetros histológicos hepáticos como la balonización, la inflamación lobular y la esteatosis. No se observó mejoría en la fibrosis cuando se examinaron todas las drogas de esta familia, pero cuando el análisis se limitó a tres estudios que usaron pioglitazona hubo una mejoría significativa en la fibrosis entre los pacientes tratados con pioglitazona en comparación con placebo (OR 1,7) 20,21,22.

\section{Incretinas}

Las incretinas, péptido similar al glucagón tipo 1 (GLP-1) y el péptido insulinotrófico dependiente de glucosa (GIP), son hormonas producidas en la mucosa intestinal que se secretan en respuesta al estímulo de nutrientes.

Sus principales acciones son: aumento de la síntesis y secreción de insulina dependiente de glucosa, incremento de la masa de células beta pancreáticas, supresión del apetito y retraso del vaciamiento gástrico. El GLP-1 además produce la inhibición de la secreción de glucagón dependiente de glucosa, disminuye la gluconeogénesis y glucógenolisis, y suprime la lipogénesis hepática mediante la activación de la proteína quinasa activada con AMP2.

El receptor de GLP-1 está presente en hepatocitos humanos y demostró tener un rol directo en la disminución de la esteatosis hepática in vivo al modular componentes de la vía de señalización de la insulina ${ }^{23}$.

Las incretinas tienen una vida media corta por la rápida degradación e inactivación que realiza la enzima dipeptidil peptidasa-4 (DPP-4). Esto condujo al desarrollo de fármacos inhibidores de la DPP-4 que influyen en la homeostasis de la glucosa al bloquear la desactivación del GLP-1 y del GIP por lo cual se emplean como tratamiento de la DM2.

\section{Agentes agonistas de los receptores del péptido similar al glucagón tipo 1 (GLP-1)}

El GLP-1 es producido por las células $L$ del intestino delgado en respuesta a los nutrientes que llegan a esa porción del tubo digestivo. Los análogos de GLP-1 ya sea de acción corta (dos veces al día) o de acción larga (diaria o semanal) mimetizan los efectos del GLP-1 endógeno sobre el páncreas al estimular la producción de insulina sumado a los efectos extrapancreáticos; a nivel del sistema nervioso central inducen saciedad y también hay neuronas productoras de GLP-1 en el núcleo del tracto solitario y se extienden a regiones del hipotálamo que son importantes en la regulación de la ingesta de alimentos; a nivel del corazón mejoran la función cardíaca, y a nivel hepático suprimen la producción hepática de glucosa independientemente del glucagón de modo que mejoran el control glucémico en los pacientes diabéticos y se obtienen buenos resultados también en el tratamiento de la obesidad $^{24}$. Algunos estudios con análogos del GLP-1 han mejorado la esteatosis hepática y descendido los niveles de enzimas hepáticas en roedores, demostrando efecto sobre receptores hepáticos en estos animales ${ }^{25}$.

El efecto de los análogos del GLP-1 sobre el hígado en la población de pacientes con DM2 se desconoce. Se evaluó el efecto de liraglutide sobre EHGNA en 43 pacientes con DM2, en los cuales la grasa hepática se midió por resonancia magnética por espectroscopia con dosis de liraglutide 
de $1,2 \mathrm{mg} / \mathrm{día}$ vs placebo antes y seis meses después. En la rama de liraglutide los pacientes perdieron hasta $4,4 \mathrm{~kg}$ de peso corporal, disminuyó hasta $2,6 \%$ de hemoglobina glicosilada A1c y se redujo el nivel de grasa intrahepática; según concluyeron los autores el liraglutide fue efectivo para disminuir el contenido graso hepático en el contexto de un descenso de peso no pudiéndose excluir que podría haber un efecto directo sobre la lipogenésis hepática ${ }^{26}$.

Actualmente se están realizando varios estudios con análogos de GLP-1 en pacientes con EHGNA con resultados promisorios, como es el caso del estudio Liraglutide Effect and Action in Diabetes 2 (LEAD) en el cual se evaluaron 3.967 adultos con el score de fibrosis (NFS) la severidad de fibrosis hepática ${ }^{27}$. Con liraglutide en dosis de $1,8 \mathrm{mg} / \mathrm{d}$ y EHGNA indicado durante 26 semanas, se observó descenso de los niveles de alanina aminotransferasa (ALT), mejoría del NFS con respecto al placebo y reducción de la progresión de la EHGNA ${ }^{28}$.

Estos estudios de fase III evaluaron los efectos hepáticos de los análogos del GLP-1 en pacientes con EHGNA y EHNA con cohortes limitados; todos refirieron en los pacientes descenso de peso, disminución de la grasa intrahepática y una tendencia a la mejoría de la fibrosis.

\section{Agentes inhibidores de la dipeptidil peptidasa 4 (DPP-4)}

Los estudios con inhibidores de la DPP-4 mostraron resultados combinados, aún así podrían ser eficaces en el tratamiento de la EHGNA ya que en algunos estudios preclínicos mostraron disminución de las transaminasas. El mecanismo de acción posiblemente sea a través de la activación de la MAPK y la downregulation de los genes implicados en la lipogénesis.

En estudios con RNM la sitagliptina no evidenció disminución de la grasa hepática, mientras que la vildagliptina mostró una reducción de TG hepáticos independiente de los cambios en el peso del órgano hepático a seis meses. La linagliptina mejora la sensibilidad a la insulina, la esteatosis y la inflamación hepática $23,29,30$.

La sitagliptina fue estudiada por Iwasaki et al. en pacientes con DM2 y EHGNA con disminución significativa de transaminasas, gamma glutamil transpeptidasa (GGT) y hemoglobina glicosilada $A 1 c^{31}$, mientras que otros autores no obtuvieron cambios significativos en las enzimas hepáticas ${ }^{32}$.

Tenegliptina, un inhibidor de la DPP-4, mejora la histopatología hepática y disminuye los niveles de TG intrahepáticos en ratones modelos, asociado a una downregulation de los genes relacionados con la lipogénesis hepática por activación de la $\mathrm{AMPK}^{33}$.

Existe evidencia creciente que las terapias basadas en incretinas tienen efectos beneficiosos sobre los hepatocitos, sin embargo se necesitan más estudios para evaluar el efecto a largo plazo sobre la EHGNA.

\section{Inhibidores del cotransportador de sodio-glucosa tipo 2 (iSGLT-2)}

Los inhibidores del cotransportador sodio-glucosa tipo 2 (iSGLT-2) representan un cambio conceptual en el tratamiento de la DM2 por su mecanismo de acción no insulinodependiente.

Los iSGLT-2 reducen la reabsorción de glucosa en el túbulo proximal causando una glucosuria de aproximadamente 60-80 g/día; esta característica los diferencia de otras drogas utilizadas en el tratamiento de la DM2 al favorecer la durabilidad de su efecto. Los efectos beneficiosos adicionales incluyen: bajo riesgo de hipoglucemia, pérdida de peso a expensas de la masa grasa y disminución de la presión arterial.

Tres fármacos de esta clase están aprobados por la Food and Drugs Administration (FDA) de los EE.UU.: dapagliflozina, canagliflozina y empagliflozina. Sin embargo, algunos otros medicamentos de esta clase -ipragliflozina, tofogliflozina y luseogliflozina- están aprobados en Japón.

Pocos estudios examinaron el impacto de los iSGLT-2 en el desarrollo de la EHGNA y/o EHNA en modelos de roedores ${ }^{34}$ con ipragliflozina y un estudio utilizó seogliflozina; todos demostraron que los iSGLT-2 atenuaron el desarrollo de EHGNA o EHNA.

Otro estudio investigó el efecto de la empagliflozina asociado a linagliptina (DPP-4) sobre la esteatohepatitis y la fibrosis en ratones con DM2. Se demostró que la empagliflozina impedía el desarrollo de la EHNA por sus efectos antiinflamatorios y anti-fibróticos, y la combinación de empagliflozina y linagliptina mejoró la EHNA sinérgicamente con efectos anti-fibróticos más fuertes.

Se ha demostrado que la empagliflozina tiene el potencial de prevenir la EHNA a través de sus acciones anti-esteatósicas y antiinflamatorias en 
un modelo de ratón de EHNA con DM2, y la combinación de empagliflozina y linagliptina sinérgicamente previno el desarrollo de fibrosis hepática lo que sugiere que podría convertirse en una nueva estrategia terapéutica en la EHNA asociada a DM2. Otros iSGLT-2 como la tofogliflozina, luseogliflozina y remogliflozina también se han indicado para mejorar las esteatohepatitis en modelos animales ${ }^{35,36}$.

En conclusión, los iSGLT-2 tienen un inmenso potencial para el tratamiento de la DM2 así como también en las enfermedades asociadas como obesidad, enfermedad cardiovascular y esteatohepatitis.

\section{Otros agentes terapéuticos estudiados para el tratamiento de la EHGNA}

\section{Orlistat}

Orlistat o tetrahidrolipstatina es una droga desarrollada para el tratamiento de la obesidad disponible en el mercado desde 1992; tiene como mecanismo de acción la inhibición reversible de la lipasa pancreática al limitar la absorción de las grasas a nivel del yeyuno-íleon. Así, el 30\% de los triglicéridos dietarios se elimina por las heces evitando su absorción ${ }^{37}$.

El orlistat para el tratamiento de la EHGNA y EHNA no parece tener efectos fármaco-específicos; se describe una mejoría de las aminotransferasas plasmáticas, de la resistencia a la insulina y de la histología hepática asociada a la pérdida de peso ${ }^{38}$.

Estudios con orlistat en dosis de $120 \mathrm{mg}$ tres veces al día y restricción calórica moderada (25 kcal/Kg de peso/d) durante seis meses mostraron una pérdida media de peso de $5,1 \mathrm{~kg}$, disminución de la esteatosis en un $71 \%$, reducción de los indicadores de inflamación del $78 \%$ y de fibrosis en un $72 \%$, así como mejoría de los niveles de transaminasas séricas, índice de resistencia a la insulina, colesterol total y triglicéridos, todos estos cambios no se correlacionaron con la pérdida de peso $^{39}$. Si bien respecto de este estudio, la falta de un brazo placebo y el uso concomitante de restricción calórica impiden sacar conclusiones sobre la efectividad del orlistat en la EHNA como intervención única.

La evaluación de los diferentes estudios publicados en su conjunto es compleja; la mayoría asocia restricción calórica y/u otros fármacos, más la evidencia que apoya la eficacia de orlistat en pacientes con EGHNA/EHNA es insuficiente sumado a que no se evaluó la mejoría en la histología hepática ${ }^{40}$.

\section{Vitamina $E$}

La vitamina E es un agente antioxidante, soluble en grasas, que se ha propuesto para mejorar los parámetros histológicos en pacientes con EGHNA/EHNA ${ }^{8}$.

Esta vitamina es una mezcla de ocho tocoferoles y ocho tocotrienoles, siendo el $\alpha$ tocoferol el componente más activo en humanos debido a su alta afinidad con la proteína transportadora de tocoferoles, el principal transportador desde el intestino al hígado. La forma sintética de la vitamina es una mezcla racémica de diferentes enantiómeros, aproximadamente un $50 \%$ más potente que la forma natural: el RRR $\alpha$ tocoferol. Las diferentes formulaciones de la vitamina $E$ pueden diferir en sus componentes y en su estructura racémica; por lo tanto cuando se comparan resultados de diferentes estudios es importante aclarar la fórmula de vitamina $\mathrm{E}$ usada ${ }^{41}$. Esta vitamina tiene efectos sobre la peroxidación de lípidos y la estabilización de las membranas, así como efectos sobre la apoptosis, la inflamación y el depósito de colágeno. Posiblemente su acción sobre PPARs, TGF $\beta 1$ (transforming growth factor $\beta 1$ ) y la regulación de genes serían los responsables de esta acción benéfica sobre la inflamación y la esteatosis hepática ${ }^{42}$.

Algunos estudios de intervención evaluaron la acción de esta vitamina. El estudio Pioglitazone versus vitamin $E$ versus placebo for the treatment of nondiabetic patients with nonalcoholic steato hepatitis trial (PIVENS) demostró que la vitamina $\mathrm{E}$ (RRR $\alpha$ tocoferol) en dosis de $800 \mathrm{UI} /$ día, comparada contra placebo, mejora la inflamación y la esteatosis hepática en un 36\% de los pacientes vs $21 \%$ en la rama placebo, pero no mejora la fibrosis en adultos con EHNA. Reduce los niveles de alanina aminotransferasa en aquellos pacientes respondedores donde también mejora la histopatología ${ }^{43}$.

El estudio Treatment of NAFLD in Children (TO$\mathrm{NIC})$ que incluyó 173 chicos y adolescentes de entre 8 y 17 años con NASH demostró superioridad de la vitamina $\mathrm{E}$ vs placebo en el balonamiento pero no en la reducción de las transaminasas hepáticas, inflamación lobular, esteatosis y fibrosis ${ }^{44}$. Aunque la vitamina $\mathrm{E}$ usada en los estudios para EHGNA/EHNA no se asoció con ningún efecto adverso mayor, deben tenerse en cuenta las dudas sobre su seguridad a largo plazo, especialmente las relacionadas con el aumento de la mortalidad como fue demostrado en un gran metaanálisis de 
135.000 pacientes provenientes de 19 estudios, aunque estos resultados no han sido demostrados por otros metaanálisis ${ }^{45}$ y el aumento de cáncer de próstata por exposición a largo plazo ${ }^{20}$.

\section{Pentoxifilina}

La pentoxifilina es un inhibidor no selectivo de la fosfodiesterasa con acción antagonista en los receptores de adenosina $2^{42}$. Se ha sugerido como una terapéutica útil debido a sus acciones agonistas sobre el factor de necrosis tumoral $\alpha$ (TNF $\alpha$ ), la reducción del stress oxidativo y los posibles efectos antifibróticos ${ }^{46}$.

La respuesta histológica se asocia con una disminución plasmática de los lípidos oxidados, sugiriendo que una disminución de la peroxidación lipídica podría tener un efecto beneficioso ${ }^{47}$, y en otro estudio en EHNA con pentoxifilina 400 mg tres veces al día vs placebo en 55 pacientes hubo mejoría histológica de la esteatosis en el 38,5\% de los pacientes comparado con el 13,5\% del grupo placebo $(p: 0,036)^{48}$. Finalmente la pentoxifilina es una droga segura, bien tolerada, que mejora la esteatosis hepática, disminuye los niveles de inflamación lobular y fibrosis pero se requieren estudios de mayor alcance para establecer la función en la EHGNA/EHNA?

\section{Drogas que disminuyen los lípidos en sangre: estatinas, fibratos, ezetimibe}

En una revisión sistemática se identificaron dos ensayos clínicos aleatorios con un número muy reducido de participantes. En el primero se comparó simvastatina vs placebo, y en el segundo atorvastatina vs fenofibrato vs una combinación de ambas drogas ${ }^{49}$.

Con simvastatina vs placebo en pacientes con EHNA no mostró efectos significativos sobre los niveles de las enzimas hepáticas o en la histología hepática. En el segundo estudio, el n fue de 186 pacientes con EHGNA y no se evidenciaron diferencias estadísticamente significativas entre ninguno de los tres grupos luego de un año de intervención en relación a las enzimas hepáticas, aspartatoaminotransferasa, la alanina-aminotransferasa, la gamma-glutamil transpeptidasa o las fosfatasas alcalinas. Aumentaron los niveles de triglicéridos, parecieron mayores en el grupo de fenofibrato comparado con el grupo de atorvastatina y no se evaluó la histología hepática ${ }^{49}$.

EI EHGNA se asoció a obesidad y DLP; las es- tatinas disminuyeron la síntesis de colesterol por inhibición de la 3-hidroxi-3-metilglutaril-coenzima A (HMG-CoA) reductasa sumado a los efectos pleiotrópicos de las estatinas que son antiinflamatorias, por lo cual se consideraron como una opción terapéutica para el hígado graso, si bien su eficacia no ha sido validada y no se encontraron pruebas para apoyar o rechazar su uso en pacientes con EHGNA/EHNA; sin embargo, la indicación de estatinas puede ser beneficiosa para mejorar el estado metabólico y reducir el riesgo de enfermedad cardiovascular.

El ezetimibe disminuye los niveles plasmáticos de colesterol por inhibición de la absorción de colesterol dietario; al combinarlo con acarbosa, un inhibidor de $\alpha$-glucosidasa, mejoró los hallazgos histopatológicos (esteatosis, inflamación y fibrosis) en un modelo de ratones de EHGNA, así como en otro estudio se evidenció disminución de las enzimas hepáticas aspartato aminotransferasa (AST) y alanina aminotransferasas (ALT), incluso del colesterol de lipoproteína de baja densidad (LDL). El seguimiento de las biopsias hepáticas reveló mejoría en el grado de esteatosis y esteatohepatitis.

Si bien los pacientes que presentaron fibrosis no manifestaron ningún cambio, en otros estudios se ha descripto que la utilización de ezetimibe a largo plazo (10 mg/día durante 24 meses) mejoró significativamente los niveles séricos de triglicéridos, colesterol total, colesterol LDL y ALT en pacientes con EGHNA y las características histológicas de la esteatosis, necroinflamación y balonización mejoraron significativamente desde la línea de base sin mejoría en la fase de fibrosis ${ }^{50}$.

\section{Ácido ursodesoxicólico}

El ácido ursodesoxicólico (UDCA) es un ácido biliar hidrófilo con propiedades antiapoptóticas y citoprotectoras. Se realizaron estudios para evaluar los efectos del UDCA en pacientes con EHGNA/EHNA cuyos resultados no mostraron beneficio significativo, ni mejora en la histología aunque sí con altas dosis manifestaron mejoría de las enzimas hepáticas y de los parámetros metabólicos si bien no se realizó evaluación histológica y se trató de un estudio de corto plazo de 12 semanas.

Al combinarlo con vitamina E la mejoría fue más evidente en los pacientes con EHNA, pero esto podría deberse solamente a la acción de dicha vitamina ${ }^{50}$. Las guías de tratamiento no indican su uso en pacientes con EHGNA/EHNA ${ }^{\text {. }}$. 


\section{Ácidos grasos poliinsaturados omega 3}

Los ácidos grasos poliinsaturados N-3 (PUFAs n-3) son ligandos de los PPAR $\alpha$, por ello se considera que podrían jugar un papel en el tratamiento de la EHGNA. Se ha visto en ratas con EHNA que la suplementación con n-3 PUFAs mejora la esteatosis hepática; en humanos los suplementos de PUFA disminuyeron significativamente en suero las enzimas hepáticas, los triglicéridos y los niveles de glucosa en pacientes con EHNA vs controles.

También se observó mejoría de la eco-textura hepática después de la suplementación con PUFA n-3 con ácido eicosapentaenoico (EPA) y docosahexaenoico (DHA) luego de un año con disminución de los niveles séricos de ALT y mejoría de la histología hepática, reducción de la esteatosis, la inflamación lobular, la balonización y la fibrosis en la mayoría de los pacientes con EHNA.

Sin embargo otros estudios demostraron regulares resultados por lo que las conclusiones y recomendaciones para la suplementación de n-3 PUFA son difíciles de establecer dado que las cantidades específicas y las proporciones de EPA y DHA no se aclararon en la mayoría de los ensayos publicados.

\section{CIRUGÍA BARIÁTRICA COMO TRATAMIENTO DE LA EHGNA}

Se ha observado mejoría histológica de la EHGNA luego de la cirugía bariátrica, con disminución de la esteatosis, la inflamación y la fibrosis ${ }^{51}$. Una revisión de Cochrane analizó 21 estudios de cohortes, prospectivos y retrospectivos, de pacientes con diagnóstico de EHGNA que fueron operados de cirugía bariátrica. La mayoría de los estudios describió mejoría en los puntajes indicadores de esteatosis e inflamación, sin embargo en cuatro estudios se detectó cierto deterioro en el nivel de fibrosis $^{52}$. La mayoría de los estudios fue con un número reducido de pacientes, no tuvieron un diseño prospectivo y los intervalos de las biopsias de control luego de la cirugía fueron variables. Por lo tanto, no pueden realizarse conclusiones definitivas para considerar a la CB como una indicación para el tratamiento de la EHGNA.

La Asociación Americana de Gastroenterología, la Asociación Americana para el Estudio de las Enfermedades Hepáticas y el Colegio Americano de Gastroenterología ${ }^{53}$ consideran a la cirugía bariátrica como una posibilidad terapéutica en pacientes obesos que tienen enfermedad hepática como comorbilidad, y sugieren que la presencia de EHGNA o de esteatosis hepática no alcohólica (EHNA) no contraindica la cirugía. Ésta podría disminuir la probabilidad de desarrollar cirrosis en pacientes con alto riesgo, sumado a la mejoría de las enfermedades asociadas como la DM2 y la enfermedad cardiovascular.

Sin embargo sería prematuro considerar a la CB como una opción terapéutica específica para la EHNA. Se requieren estudios con mayor número de pacientes, con seguimiento a más largo plazo y con grupos de pacientes comparables en grado de severidad de la enfermedad hepática y las comorbilidades asociadas ${ }^{53}$. La enfermedad hepática es heterogénea y puede estar presente en personas sin obesidad ni otros componentes del síndrome metabólico ${ }^{54}$. Aún no está del todo claro si debe considerarse la realización de la CB con el objetivo de mejorar el hígado graso o la esteatohepatitis, tampoco cuál sería la mejor técnica quirúrgica. No existen hasta el momento estudios aleatorizados controlados que evalúen cierta técnica quirúrgica vs intervenciones en el estilo de vida, placebo o fármacos, o de una técnica quirúrgica vs otra en la resolución de la esteatohepatitis. La mayoría de los estudios disponibles no es prospectivo y en varios las biopsias de control postquirúrgico se realizaron luego de intervalos variables de tiempo siguientes a la cirugía, con lo cual los resultados podrían no ser comparables.

Con respecto a los beneficios de la CB sobre la EHGNA no se sabe si pueden atribuirse exclusivamente al descenso de peso, o si la cirugía produce un efecto metabólico intrínseco sobre el hígado. Las trayectorias en el descenso de peso difieren según las técnicas quirúrgicas y podrían tener impacto en las consecuencias sobre el hígado, mientras que un descenso de peso moderado de aproximadamente el 5-7\% puede ser suficiente para mejorar la esteatosis; es necesario un descenso mayor al 8-10\% para mejorar la esteatohepatitis y una reducción de peso mayor al $10 \%$ para producir una regresión significativa de la fibrosis. Las cirugías malabsortivas podrían empeorar transitoriamente la función así como la histología hepática en los primeros seis meses con normalización posterior de ambas a los 12 meses $^{55}$. Con el descenso de peso significativo que se alcanza luego de la CB se han descripto reducciones del 90\% de la esteatosis hepática, $80 \%$ de la esteatohepatitis y disminución incluso de la fibrosis en un $65 \%$. Este dato se confirmó en un estudio pros- 
pectivo reciente en el cual aproximadamente el $50 \%$ de los pacientes experimentó mejoría en la puntuación de indicadores de fibrosis luego de la cirugía ${ }^{56}$. La CB redujo la mortalidad en comparación con el grupo control (HR: 0,54), pero a los 10 años de seguimiento este efecto no se mantuvo en los pacientes que presentaban esteatohepatitis al momento de la cirugía. Se necesitan más estudios para evaluar si los resultados sobre la mortalidad se asocian o no a la persistencia de la DM2 luego de la cirugía. Aunque debe mencionarse que las muertes observadas se debieron a sepsis y no tuvieron relación directa con la enfermedad hepática ${ }^{57}$.

En otro estudio prospectivo en el que se incluyeron 109 pacientes con obesidad mórbida y EHNA comprobada por histología al momento de la cirugía bariátrica, se obtuvo biopsia hepática de control al año de seguimiento. Se observó la desaparición de EHNA en 92 pacientes (85\%), en 94\% de los que presentaba EHNA leve-moderada vs $70 \%$ de los que tenía enfermedad severa. La esteatosis disminuyó en el $60 \%$, el balonamiento hepatocelular se redujo en el $84 \%$ de las muestras, la inflamación lobular en el $67 \%$ y la fibrosis se redujo en el $33,8 \%$ de los pacientes. Los sujetos intervenidos con banda gástrica ajustable tuvieron mayor persistencia de EHNA $(30,4 \%)$ vs los operados de bypass gástrico en $Y$ de Roux (BGYR) (1,6\%).

Un metaanálisis de 15 estudios y 766 pares de biopsias mostró resolución o mejoría de la esteatosis en el 91,6\% de los casos, de la esteatohepatitis en el $81,3 \%$ y de la fibrosis en el $65,5 \%$. Se observó resolución completa de la esteatohepatitis en el $69,5 \%$ de los pacientes ${ }^{58}$.

En el Gráfico 1 se observa el cambio en el grado de actividad inflamatoria antes de la cirugía y un año después ${ }^{56}$. La escala Metavir es una de las más utilizadas para evaluar las fases de la enfermedad, donde estadio 0 (F0) describe ausencia de fibrosis, estadio 1 (F1) fibrosis leve, estadio 2 (F2) fibrosis moderada, estadio 3 (F3) fibrosis grave y estadio 4 (F4) cirrosis. También se describe mejoría de la fibrosis con la escala Metavir en el Gráfico 2.

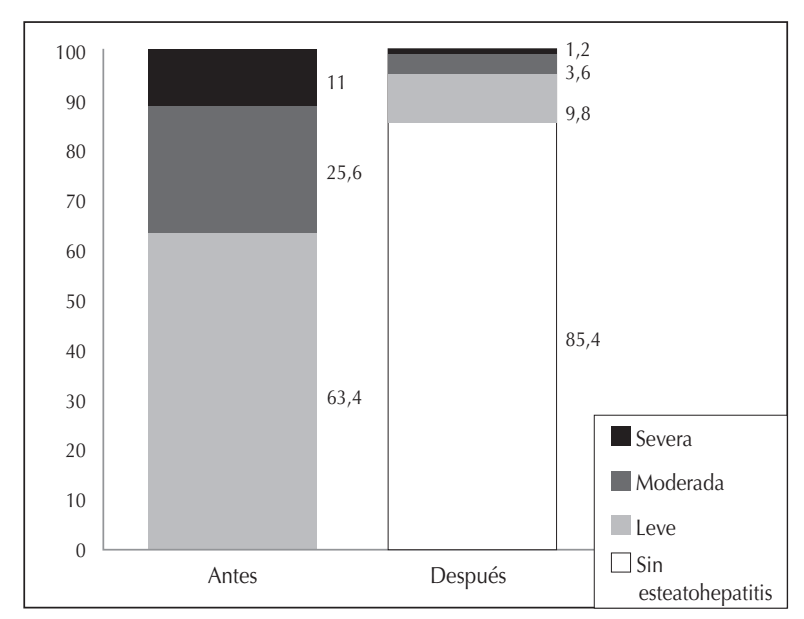

Gráfico 1: Actividad inflamatoria al inicio y al año después de la cirugía (Adaptado de Lassailly et al.)

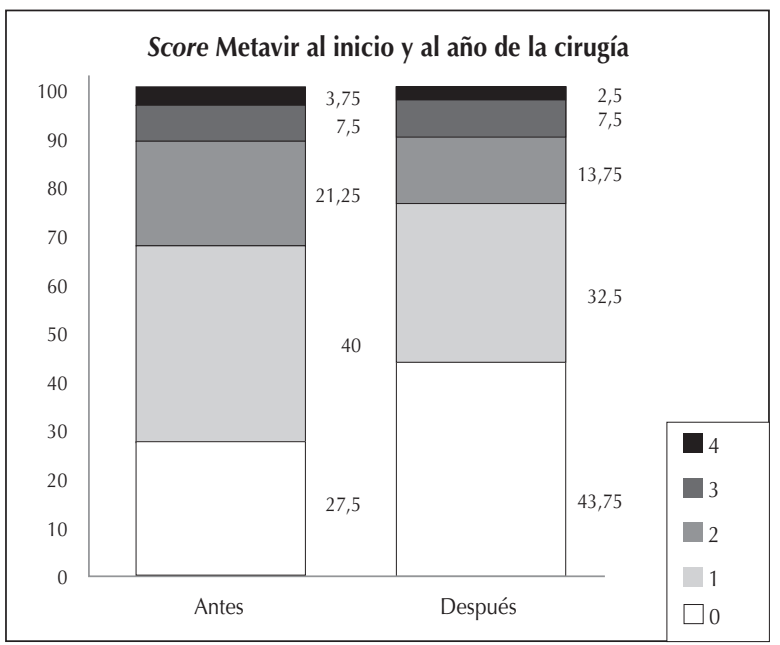

Gráfico 2: Escala Metavir (Adaptado de Lassailly et al.)

\section{Mecanismos de mejoría de la inflamación hepática}

Los mecanismos a través de los cuales la cirugía bariátrica mejora la EHGNA y la EHNA son complejos y se encuentran todavía en estudio. Se plantean como mecanismos posibles de la mejoría de la EHNA en pacientes sometidos a bypass gástrico en Y de Roux (BGYR) la mejoría de la insulinorresistencia y del metabolismo lipídico, la disminución de las citoquinas inflamatorias implicadas en la patogenia de la inflamación y la fibrosis como la interleuquina 6 (IL-6), el factor de necrosis tumoral- $\alpha$ (TNF $\alpha$ ), la proteína quimiotáctica de monocitos 1 (MCP-1), el factor de crecimiento transformante- $\beta$ (TGF- $\beta$ ), el factor de crecimiento endotelial vascular (VEGF), del angiotensinógeno y la angiotensina II, y de otros factores fibrogénicos en la regulación de la fibrosis ${ }^{59}$. 
Los mecanismos a través de los cuales la cirugía bariátrica mejora la EHGNA y EHNA son complejos y todavía en estudio. La cirugía bariátrica actúa sobre diferentes niveles: las hormonas gastrointestinales, la inflamación, la dislipidemia, la insulinorresistencia y la obesidad. Una síntesis de los mecanismos propuestos se detalla en el Gráfico $3^{51}$.

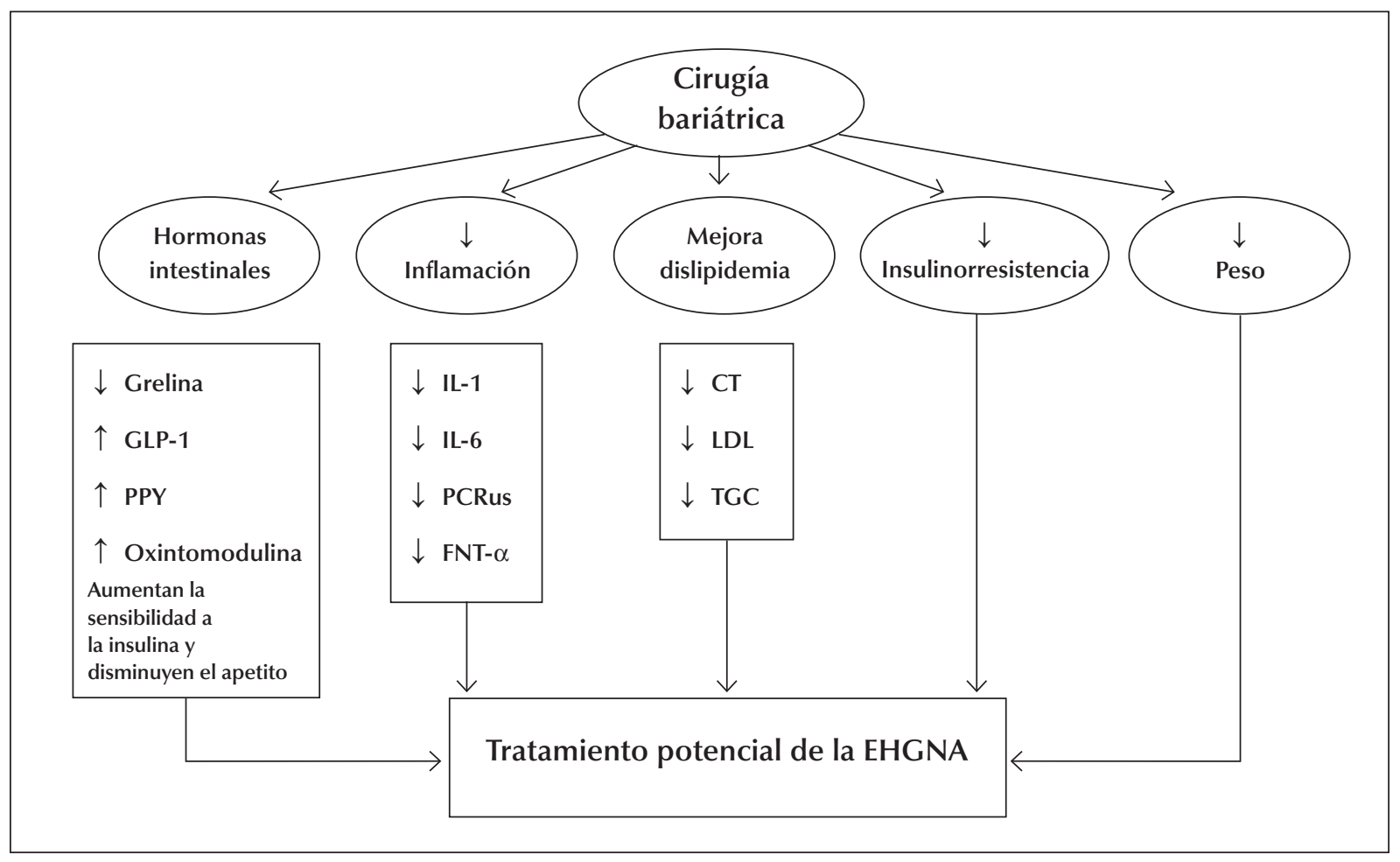

Gráfico 3: Mecanismos de acción de la CB en la EHGNA (Adaptado de Hassef et al.).

\section{Resultados de la CB sobre la EHGNA}

En 160 pacientes operados de CB, con biopsia inicial y nueva toma de muestra alrededor de los 30 meses del postoperatorio, se observó resolución de la esteatosis en el $75 \%$ de los casos, de la esteatohepatitis en el $90 \%$ y regresión o mejoría de la fibrosis en el $60 \%$ de los pacientes ${ }^{54}$.

Mathurin et al. ${ }^{60}$ realizaron una toma de biopsia hepática a 362 pacientes antes, al año y cinco años después de la CB. Mostraron disminución significativa de la esteatosis hepática, cuya mejoría estuvo íntimamente relacionada con la disminución de la insulinorresistencia. El porcentaje de pacientes con esteatohepatitis disminuyó de 27,4 a 14,2\%. Hubo un leve aumento de la fibrosis, aunque en el $95 \%$ de los casos fue con puntuación igual o menor a 1 (rango de 0-3). Los beneficios más importantes se observaron al año y persistieron a los cinco años. Es de notar que los pacientes que presentaron fibrosis luego de la cirugía tenían IMC más alto previo a la misma y mayor grado de resistencia a la insulina. Podría inferirse que la peor evolución se relaciona con la historia natural de la enfermedad más severa, que por una consecuencia misma de la cirugía o de la técnica aplicada. La mejoría de la esteatosis y del balonamiento hepatocelular evolucionó en paralelo con la mejoría de la insulinorresistencia, independientemente del IMC. Estos datos pueden extrapolarse para el daño sobre otras vísceras asociado a la insulinorresistencia ${ }^{59}$.

Un estudio de pacientes con sobrepeso y obesidad evaluó la efectividad y el costo-beneficio de la CB y la interpretó de acuerdo al grado de fibrosis hepática. En sus conclusiones refiere que la cirugía mejora la calidad de vida de todos los pacientes, siendo costo-efectiva en pacientes con obesidad independientemente del grado de fibrosis. En pacientes que sólo presentan sobrepeso resultaría costo-efectiva en aquellos con fibrosis avanzada (F-3 en escala de 0-4, considerando que los pacientes con F4 son candidatos a trasplante hepático $)^{53}$. 
Existen escasos datos sobre CB en pacientes con cirrosis. Los pacientes con cirrosis tienen un riesgo perioperatorio más alto, además de asociarse con patología metabólica y cardiovascular que de por sí aumentan la morbimortalidad. Se estima que el diagnóstico de cirrosis no conocida hasta el momento de la cirugía llega al 1,4\% de los pacientes operados por cualquier causa. La mortalidad perioperatoria con $\mathrm{CB}$ en pacientes con cirrosis es de 1,2 vs $0,3 \%$ en pacientes sin esa condición ${ }^{61}$.

Un estudio de la Cleveland Clinic muestra los resultados de la intervención con cirugía bariátrica en 23 pacientes con cirrosis, 12 (52\%) con cirrosis conocida previamente y $11(48 \%)$ no conocidas hasta el momento de la cirugía, con un IMC promedio de $48 \mathrm{~kg} / \mathrm{m}^{2}$ y edad media de 51 años, $14(61 \%)$ eran mujeres, 19 pacientes $(83 \%)$ tenían diagnóstico de DM2 e HTA, y 14 (61\%) presentaban dislipidemia. Fueron seguidos a mediano plazo, con buenos resultados en cuanto al descenso de peso y la mejoría de las comorbilidades, lo que muestra que en centros seleccionados y con los cuidados adecuados la CB puede realizarse aún en estos pacientes ${ }^{62}$.

Con la técnica quirúrgica de bypass íleo-yeyunal, que se realizó entre las décadas de 19601970, en algunos pacientes se describieron progresión de la fibrosis e insuficiencia hepática. Esa técnica ha caído en desuso y fue reemplazada por técnicas más seguras. Con el BGYR se observó leve progresión a la fibrosis en pacientes que respondieron pobremente a la CB con descenso de peso insuficiente. La peor evolución de la fibrosis podría deberse a la historia natural más severa de la enfermedad, con menos beneficios alcanzados con la CB y podría no deberse a una complicación causada por la cirugía. Sin embargo, otros estudios muestran reducción de la fibrosis y de los procesos necro-inflamatorios que pueden prevenir el desarrollo de cirrosis y sus complicaciones ${ }^{63}$.

Podemos concluir que si bien muchos estudios muestran la mejoría de la EHGNA en pacientes sometidos a $\mathrm{CB}$, se requieren aún más estudios clínicos randomizados controlados para obtener una evaluación definitiva de los beneficios y los daños de la CB como un enfoque terapéutico dirigido a los pacientes con EHGNA.

Finalmente resumimos las recomendaciones de tratamiento propuestas en las Guías de Práctica Clínica para el Manejo de la Enfermedad Hepática Grasa No Alcohólica ${ }^{8}$ (EHGNA) según ca- tegoría de evidencia publicadas ${ }^{8}$ por la European Association for the Study of the Liver (EASL), la European Association for the Study of Diabetes (EASD) y la European Association for the Study of Obesity (EASO) en el año 2016.

\section{Recomendaciones en el cambio de estilo de vida}

- Se aconsejará un programa estructurado de cambio de estilo de vida que incluya dieta saludable y actividad física habitual. Categoría C2.

- Los pacientes sin EHNA o fibrosis sólo deberán recibir consejería sobre dieta saludable y actividad física, y no debería indicarse farmacoterapia. Categoría B2.

- En pacientes con sobrepeso/obesidad con EHGNA, el descenso del 7-10\% del peso corporal con intervención en el estilo de vida mejorarán las enzimas hepáticas y la histología. Categoría B1.

- Las recomendaciones dietéticas podrán considerar restricción calórica excluyendo alimentos que promuevan la EHGNA como bebidas con alto contenido de fructosa. La dieta deberá ajustarse en lo posible, en cuanto a los macronutrientes, a una dieta mediterránea. Categoría B1.

- Los ejercicios aeróbicos como los anaeróbicos son beneficiosos para reducir la grasa hepática. La elección debe basarse en la preferencia de los pacientes para asegurar su mantenimiento en el largo plazo. Categoría B2.

\section{Recomendaciones farmacológicas}

- La farmacoterapia debe reservarse sólo para pacientes con EHNA, particularmente los que presenten fibrosis significativa (estadio 2 ó más). En los pacientes con enfermedad de menor severidad pero alto riesgo de progresión (por ejemplo, diabéticos con aumento persistente de ALT o alta proporción de necroinflamación) puede indicarse medicación para prevenir la progresión de la enfermedad. Categoría B1.

- Pueden indicarse para la EHNA, si bien no hay recomendación firme, la pioglitazona (mayor eficacia si bien fuera de etiqueta ya que es una droga para el tratamiento de la DM2) o la vitamina $E$ (mayor seguridad y tolerabilidad en el corto plazo) o su combinación. Categoría B2.

- La duración óptima del tratamiento se desconoce; si no hay reducción de ALT a los seis 
meses de tratamiento deberá suspenderse el mismo. En pacientes con cifras basales normales no pueden hacerse recomendaciones con respecto a fármacos. Categoría C2.

- Las estatinas pueden utilizarse con confianza para reducir el LDL-colesterol y prevenir el riesgo cardiovascular, sin beneficios o daños en la enfermedad hepática. Los ácidos grasos poliinsaturados n-3 reducen el plasma y los lípidos hepáticos, pero no hay datos que apoyen su uso específicamente para la EHNA. Categoría B1.

\section{Recomendaciones para la cirugía bariátrica}

- Al mejorar la obesidad y la diabetes, la cirugía bariátrica (metabólica) reduce la grasa hepática y es probable que disminuya la progresión de EHNA; los datos prospectivos demostraron una mejoría en todas las lesiones histológicas de EHNA, incluida la fibrosis. Categoría B1.

\section{BIBLIOGRAFÍA}

1. Quintero F, Botero V, D’Agostino D et al. Enfermedad del hígado graso no alcohólico (EHGNA): revisión y puesta al día. Grupo de Trabajo de la Sociedad Latinoamericana de Gastroenterología Hepatología y Nutrición Pediátrica (SLAGHNP). Acta Gastroenterol Latinoam 2016; 46: 246-264.

2. Bril F, Cusi K. Nonalcoholic fatty liver disease. The new complication of type 2 diabetes mellitus. Endocrinol Metab Clin N Am 2016; 45:765-781.

3. Della-Corte C, Mazzotta A, Nobili V. Enfermedad del hígado graso y obesidad en pacientes jóvenes. Curr Opin Endocrinol Diabetes and Obes 2016; 23:66-71.

4. Vernon G, Baranova A, Younossi Z. Systematic review: the epidemiology and natural history of non-alcoholic fatty liver disease and non- alcoholic steaohepatitis in adults. Alim Pharamacol Ther 2011; 34: 274-285.

5. Younossi Z, Koening A, Abdelatif D, Fazel Y, Henry L, Wymer M. Global epidemiology of nonalcoholic fatty liver dieases. Metaanalytic assessment of prevalence, incidence, and outcomes. Hepatology 2016; 64 (1): 73-84.

6. Anderson P, Gual A, Colon J. Alcohol y atención primaria de la salud: informaciones clínicas básicas para la identificación y el manejo de riesgos y problemas. Washington, D.C.: OPS ${ }^{\odot} 2008$ ISBN 9789275328569.

7. Cusi K. Treatment of patients with type 2 diabetes and non-alcoholic fatty liver disease: current approaches and future directions. Diabetología 2016; 59: 1112-1120.

8. European Association for the Study of the Liver (EASL), European Association for the Study of Diabetes (EASD) and European Association for the Study of Obesity (EASO). Clinical Practice Guidelines for the management of non-alcoholic fatty liver disease. Diabetología 2016; 59: 1121-1140.

9. Kleiner D, Brunt E, Van Natta M, et al. Design and validation of a histoligical scoring system for nonalcoholic fatty liver disease. Hepatology 2005; 41:1313-1321.
10. Mottin C, Moretto M, Padoin AV, et al. Histological behavior of hepatic steatosis in morbidly obese patients weight loss induced by bariatric surgery. Obesity Surgery 2005; 15 (6): 788-793.

11. Buchwald $H$, et al. Bariatric surgery: a systematic review and meta-analysis. JAMA 2004; 292:1724-1737.

12. Hannah WN Jr, Harrison S. Effect of weight loss, diet, exercise and bariatric surgery on nonalcoholic fatty. Clin Liver Disease 2016; Vol 20 (2): 339-350.

13. Milic S, Mikolasevic I, Krznaric I, et al. Nonalcoholic steatohepatitis: emerging targeted therapies to optimize treatment options. Drug Design, Development and Therapy 2015; 9: 4835-4845.

14. Baran B, Akyüz F. Non-alcoholic fatty liver disease: What has changed in the treatment since the beginning? World J Gastroenterol 2014; 20(39): 14219-14229.

15. Musso G, Cassader M, Rosina F, et al. Impact of treatments on liver disease, glucose metabolism and cardiovascular risk in nonalcoholic fatty liver disease (NAFLD): a systematic review and meta analysis of randomised trials. Diabetologia 2012; 55: 885-9.

16. Abidin-Ozturk $Z$, Kadayifci $A$. Insulin sensitizers for the treatment of non-alcoholic fatty liver disease. World J Hepatol 2014; 6 (4): 199-206.

17. Chalasani N, Younossi Z, Lavine JE, Diehl AM, Brunt EM, Cusi K, Charlton M, Sanyal AJ. The diagnosis and management of nonalcoholic fatty liver disease: practice Guideline by the American Association for the Study of Liver Diseases, American College of Gastroenterology, and the American Gastroenterological Association. Hepatology 2012; 55(6):2005-2023.

18. Ratziu V, Bellentani S, Cortez-Pinto H, Day C, Marchesini G. A position statement on NAFLD/NASH based on the EASL 2009 special conference. J Hepatol 2010; 53: 372-384.

19. Zheng J, Woo S, Hu X, et al. Metformin and metabolic diseases: a focus on hepatic aspects. Front Med 2015; 9(2): 173-186.

20. Cusi K, Yau H, Rivera K, Lomonaco R. The future of thiazolidinedione therapy in the management of type 2 diabetes mellitus. Curr Diab Rep 2013; 13:329-341.

21. Belfort R, Harrison SA, Brown K, Darland C, Finch J, Hardies J, et al. A placebo controlled trial of pioglitazone in subjects with nonalcoholic steatohepatitis. N Engl J Med 2006; 355:2297-307.

22. Boettcher E, Csako G, Pucino F, et al. Meta-analysis: pioglitazone improves liver histology and fibrosis in patients with non-alcoholic steatohepatitis. Alimentary Pharmacology \& Therapeutics 2012; 35 (1): 66-75.

23. Klein T, Mark M, Schuppan D, et al. The dipeptidyl peptidase 4 inhibitor linagliptin is an effective therapeutic for metabolic liver disease in several rodent models of non-alcoholic fatty liver disease (NAFLD) and non-alcoholic. Hepatology 2011; 54 (4) Suppl: 36

24. Hazlehurst J, Woods C, Marjot TH, et al. Non-alcoholic fatty liver disease and diabetes. Metabolism Clinical and Experimental 2016; 65: 1096-1108.

25. Ding X, Saxena N, Lin S, et al. Exendin-4, a GLP-1 receptor agonist, reverses hepatic steatosis in ob/ob mice. Hepatology 2006; 43: 173-81.

26. Verges B, Bouillet B, Guiu B, et al.Treatment with liraglutide leads to an important reduction in liver fat content, assessed by magnetic resonance spectroscopy in people with type 2 diabetes. $\mathrm{Cli}$ nicalTrial Registration Number: EudraCT: 2012-000375-16.

27. Treeprasertsuk S, Björnsson E, Enders F, et al. NAFLD fibrosis score: a prognostic predictor for mortality and liver complications among NAFLD patients. World J Gastroenterol 2013; 19 (8): 1219-1229. 
28. Armstrong MJ, Gaunt P, Aithal G, et al. Liraglutide safety and efficacy in patients with non-alcoholic steatohepatitis (LEAN): a multicentre, double-blind, randomised, placebo-controlled phase 2 study. Lancet 2016; 387: 679-690.

29. Kern $\mathrm{M}$, Klöting $\mathrm{N}$, Niessen $\mathrm{H}$, et al Linagliptin improves insulin sensitivity and hepatic steatosis in diet-induced obesity. Plos one $2012 ; 7(6): \mathrm{e} 38744$.

30. Klein T. Linagliptin alleviates hepatic steatosis and in ammation in a mouse model of nonalcoholic. Med Mol Morphol 2014; 47:137-149.

31. Iwasaki $\mathrm{T}$, Yoneda $\mathrm{M}$, Inamori $\mathrm{M}$, et al. Sitagliptin as a novel treatment agent for non-alcoholic fatty liver disease patients with type 2 diabetes mellitus. Hepatogastroenterology 2011; 58:2103-2105.

32. AraseY, KawamuraY, SekoY, et al. Efficacy and safety in sitagliptin therapy for diabetes complicated by non-alcoholic fatty liver disease. Hepatology 2013; 43:1163-1168.

33. Takayasu I, Yohei S, Tsuneiyuki M, et al. The dipeptidyl peptidase-4 inhibitor teneligliptin attenuates hepatic lipogenesis via AMPK activation in non-alcoholic fatty liver disease model mice. Int J Mol Sci 2015; 16: 29207-29218.

34. Tahara A, Kurosaki E, Yokono M, et al. Effects of SGLT2 selective inhibitor ipragliflozin on hyperglycemia, hyperlipidemia, hepatic steatosis, oxidative stress, inflammation, and obesity in type 2 diabetic mice. Eur J Pharmacol 2013; 715:246-55.

35. Nakano S, Katsuno K, Isaji M, et al. Remogliflozin etabonate improves fatty liver disease in diet-induced obese male mice. J Clin Exp Hepatol 2015; 5 (3), 190-198.

36. Suzuki M,Takeda M, Kito A, et al. Tofogliflozin, a sodium/glucose cotransporter 2 inhibitor, attenuates body weight gain and fat accumulation in diabetic and obese animal models. Nutr Diabetes 2014; 4 (7), e125.

37. Harrison SA, Fecht W, Brunt EM, et al. Orlistat for overweight subjects with nonalcoholic steatohepatitis: a randomized, prospective trial. Hematology 2009; 49: 80-86.

38. Barb D, Portillo-Sánchez P, Cusi K. Pharmacological management of nonalcoholic fatty disease. Metabolism Clinical and Experimental 2016; 65:1183-1195.

39. Assy N, Hussein O, Abassi Z. Weight loss induced by orlistat reverses fatty infiltration and improves hepatic fibrosis in obeses patients with non-alcoholic steatohepatitis. Gut 2007; 56: 443-444.

40. Younossi ZM, Reyes MJ, Mishra A, et al. Systematic review with meta-analysis: non-alcoholic steatohepatitis- a case for personalized treatment based on pathogenic targets. Aliment Pharmacy There 2014; 39: 3-1.

41. Rotman Y, Sanyal A. Current and upcoming pharmacotherapy for non alcoholic fatty liver disease. Gut 2017; 66:180-190.

42. Mintziori G, Polyzos S. Emerging and future therapies for nonalcoholic steatohepatitis in adults. Expert Opinion on Pharmacotherapy 2016; 17:1937-1946.

43. Sanyal A, Chalasani N, Kowkley K, et al. Pioglitazone, vitamin E or placebo for nonalcoholic steatohepatitis. N Engl J Med 2010; 362:1675-1685.

44. Lavine J, Schwimmer J, Van Natta M, et al. Effect of vitamin E or metformin for treatment of nonalcoholic fatty liver disease in children and adolescent: the TONIC randomized controlled trial. JAMA 2011; 305:1659-1668.

45. Miller E, Pastor-Barriuso R, Dalal D, et al. Meta-analysis high dosage vitamin $E$ supplementation may increase all cause mortality. Ann Intern Med 2005; 142:37-46.

46. Rotman Y, Sanyal A. Current and upcoming pharmacotherapy for non alcoholic fatty liver disease. Gut 2017; 66:180-190.
47. Zein C, Lopez R, Fu X, et al. Pentoxifylline decreases oxidized lipid products in nonalcoholic steatohepatitis: new evidence on the potential therapeutic mechanism. Hepatology 2012; 56:12911299.

48. Zein C, Yerian L, Gogate P, et al. Pentoxifylline improves nonalcoholic steatohepatitis: a randomized placebo-controlled trial. Hepatology 2011; 54:1610-1619.

49. Eslami L, Merat S, Malekzadeh R, et al. Statins for non-alcoholic fatty liver disease and non-alcoholic steatohepatitis. Cochrane Database of Systematic Reviews 2013; Issue 12. Art. $N^{\circ}$ CD008623.

50. Takahashi $Y$, Sugimoto $\mathrm{K}$, Inui $\mathrm{H}$, et al. Current pharmacological therapies for nonalcoholic fatty liver disease /nonalcoholic steatohepatitis. World J Gastroenterol 2015; 21 (13): 3777-3785.

51. Hafeez S, Ahmed M. Review article bariatric surgery as potential treatment for nonalcoholic fatty liver disease: a future treatment by choice or by chance? J Obes 2013; Article ID 839275.

52. Chávez-Tapia NC, Tellez-Avila FI, Barrientos-GutiérrezT. Bariatric surgery for non-alcoholic steatohepatitis in obese patients. Cochrane Database Syst Rev 2010; (1):CD007340.

53. Chalasani N, Younossi Z, Lavine JE, et al; American Gastroenterological Association; American Association for the Study of Liver Diseases; American College of Gastroenterology. The diagnosis and management of nonalcoholic fatty liver disease: practice guideline by the American Gastroenterological Association, American Association for the Study of Liver Diseases, and American College of Gastroenterology. Gastroenterology 2012; 142:1592-1609.

54. Bril F, Cusi K. Management of nonalcoholic fatty liver disease in patients with type 2 diabetes: a call to action. Diabetes Care 2017; 40:419-430.

55. Taitano A, Markow M, Finan JE, et al. Bariatric surgery improves histological features of nonalcoholic fatty liver disease and liver fibrosis. J Gastrointest Surg 2015; 19:429-437.

56. Lassailly G, Caiazzo R, Buob D, et al. Bariatric surgery reduces features of non-alcoholic steatohepatitis in morbidly obese patients. Gastroenterology 2015; 149:379-388.

57. Goossens N, HoshidaY, Min Song W, et al. Nonalcoholic steatohepatitis is associated with increased mortality in obese patients undergoing bariatric surgery. Clinical Gastroenterology and $\mathrm{He}$ patology 2016; 14:1619-1628.

58. Mummadi R, Kasturi K, Chennareddygari S, Sood G. Effect of bariatric surgery on non-alcoholic fatty liver disease: systematic review and meta-analysis. Clinical Gastroenterology and Hepatology 2008; 1396-1402.

59. Klebanoff M, Corey K, Chhatwal J, et al. Bariatric surgery for non-alcoholic steatohepatitis: a clinical and cost-effectiveness analysis. Hepatology Online 27/2/2017; doi: 10.1002/hep.28958.

60. Mathurin P, Hollebecque A, Arnalsteen L, et al. Prospective study of the long-term effects of bariatric surgery on liver injury in patients without advanced disease. Gastroenterology 2009; 137:532-540.

61. Mosko JD, Nguyen GC. Increased perioperative mortality following bariatric surgery among patients with cirrhosis. Clin Gastroenterol Hepatol 2011; 9:897-901.

62. Shimizu H, Phuong V, Maia M, Kroh M, Chand B, Schauer PR, Brethauer SA. Bariatric surgery in patient swith liver cirrosis. Surgery for Obesity and Related Diseases 2013; 9: 1-6.

63. Grimm IS, SchindlerW, Haluszka O. Steatohepatitis and fatal hepatic failure after biliopancreatic diversion. Am J Gastroenterol 1992; 87:775-779. 